\title{
Matching Interaction Design Principles and Integrated Navigation Systems in an Electronic Classroom
}

\section{Sanja Bauk ${ }^{a}$, Silvia Fajardo-Flores ${ }^{b}$}

This article deals with cross-cultural and cross-disciplinary virtual engagement aimed to match human-computer interaction design principles and contemporary integrated navigation information systems. As interaction design principles ten general principles - heuristics are used, and as examples of contemporary navigation information systems, chart Radio Detection (or Direction) and Ranging (RADAR) device and Electronic Chart Display and Information System (ECDIS) are used. This crossdisciplinary research has been achieved through a Collaborative Online International Learning (COIL) project by Durban University of Technology (DUT) in South Africa and University of Colima (UoC) in Mexico. Namely, the students from both sides guided

\section{KEY WORDS}

$\sim$ Collaborative Online International Learning (COIL)

$\sim$ Software usability

$\sim$ Interaction design principles

$\sim$ Chart radar

$\sim$ ECDIS by their lecturers have been engaged in analyzing today's most frequently used marine navigational aids from the perspective of their reliability and user centeredness. The complexity of the systems and the lack of standards are observed as main problems when it comes to marine information systems effectiveness and their user centeredness.

\section{INTRODUCTION}

Internet has considerably changed people's lifestyle and working conditions worldwide. In parallel, modes of generating and transferring knowledge have been changing. Thanks to the technological advancements, it becomes possible to learn and lecture through a variety of channels that combine faceto-face and virtual engagements. One of those channels is the Collaborative Online International Learning (COIL). In 2014, the Durban University of Technology (DUT) became the first African institution involved in the global network of partners established at the State University of New York (SUNY) COIL system. This has contributed to the development of the curriculum, education in general and graduate attributes, provided systemic benefits for the staff and students, developed innovative cost-effective strategies (Pillay and Samuels, 2016), etc. At present, DUT has several ongoing COIL projects merging several different disciplines: medicine and journalism, chemistry and information technologies, navigational information systems and software testing methods, etc., as a result of collaboration with several overseas universities. The last mentioned couple of modules, i.e. navigational information systems (Maritime Studies Department, DUT) and software testing methods (Engineering Department, $\mathrm{UoC}$ ) within the COIL are in the focus of our research study. In 
this regard, the rest of the paper is organized as follows: Chapter 2 gives a short literature review in the field; Chapter 3 gives an overview of contemporary navigation information systems with an emphasis on chart radar and ECDIS; Chapter 4 contains basic description of human-computer interaction design principles; Chapter 5 gives applied methodology overview; the sixth part presents the outcomes achieved through applying interaction design principles upon selected navigation information systems along with the following discussion; the last chapter contains some conclusion remarks.

\section{LITERATURE REVIEW}

Several studies on the Collaborative Online International Learning (COIL) projects have been done. Here are presented some of the most comprehensive ones available to the authors. The first one deals with the social media as advanced electronic tools used to support learning among nursing and midwifery students (O'Connor et al., 2018). The key findings of this study are that social media can aid acquiring knowledge and skills among nursery and midwifery students, enhancing confidence, and facilitating professional and personal networks. It has also been concluded that the social media (Facebook, Twitter, and YouTube) as dynamic, interactive online environments can affect the learning processes in several ways. After the detailed and in-depth study, the conclusion has been reached that more robust studies are required to objectively measure whether and how social media improve learning. The second comprehensive study taken into consideration was performed at the University of Arizona, USA (Brooks and Pitts, 2016). It deals with the issue of how students consider and display their own identities through cross-cultural virtual engagement. This study has been focused on the USA college students who have participated in an array of online conversations with students from Singapore, and their conceptions on themselves relative to others in crosscultural conversations. The study leads to a conclusion that the globally-connected classroom will become a natural extension for many universities. Therefore, more critical discussions and reflections about practices and behaviors of students in their Internet-based cross-cultural exchanges are to be conducted around the globe. Within the context, it is also worth to mention the study which considers transforming teaching, learning, and research through practicing mindfulness and action research study (Brendel and Cornett-Murtada, 2019). The study fosters more mindful university culture through including mindful grading and assessment, awareness of students in the classroom, and cultivating self-awareness in teaching. The COIL projects can support the innovative approach to teaching, learning, and conducting research based on mindfulness mediation and transformative pedagogy. This kind of virtual engagement aims at internationalization of students' learning experience and their preparation for a competitive international labor market and workplace (Anderson et al., 2010; Brooks, 2011, 2012). Collaboration in multi-cultural environments across disciplines requires a higher level of mindfulness in teaching, team work, research, and providing the accompanying services. Concerning the COIL environment, learning processes and outcomes, it is also important to mention the Substitution Augmentation Modification Redefinition(SAMR) model developed by Puentedura (2006). This model has two roles in technology-supported education: enhancement and transportation. Enhancement includes substitution, where technology acts as a direct tool substitute with no functional change, and augmentation, where technology acts as a direct tool substitute with functional improvement. The examples might be a word processor used as a typewriter (substitution), and word processing with spell check (augmentation). On the other hand, transformation includes modification, where technology allows significant task redesign and redefinition, previously inconceivable. For instance, technology enables lesson redesign by using graphs, images, spreadsheets, etc., (i.e. modification), and creation of new tasks, previously inconceivable, e.g. Skype with experts, comparing and combining results via wikis and blogs, publishing worldwide online, coming up to the new conclusions by using different simulation software tools, etc., (i.e. redefinition). Hamilton et al. (2016) gave a critical review and suggestions for the use of SAMR model through three different perspectives: lack of context, rigid hierarchical structure, and putting product over process. Among others, this review concludes that it is an imperative for lecturers to understand how to use technology to promote student learning and achievement in today's interconnected world and ubiquity of technology. Lecturers should explore possibilities of how to operate effectively and efficiently in tandem with technology, in order to promote students' growth and achievement (Koehler et al., 2014). Bower et al. (2012) gave a framework for Web 2.0 learning design in the context of Technological Pedagogical and Content Knowledge (TPACK) model. In this study, the authors considered possibilities of using social bookmarking, wikis, shared documents, blogs, microblogging, presentation tools, podcasting, screen recording, mind-mapping, digital storytelling, etc., in creating a more engaging educational content. Bauk and Radlinger (2013) compared features of Windows Movie Maker, Camtasia Studia, Adobe Premiere, and other advanced dedicated software in reviving instructional materials and making them more alluring for the students. Furthermore, Bauk (2019) proposed a non-monetary return of investment model for assessing success of two recently accomplished COIL projects at DUT, through which she confirmed the hypothesis of high level of lecturers' and students' satisfaction with the virtual engagement, achieved learning outcomes and opportunities for extending further collaboration despite the impediments inherent to the virtual engagement. Recently, Jahnke (2020) has argued that 
lecture-based learning should be replaced by action-based learning and gives the following example: a group of students in the history class developed an app that virtually teaches the users about history surrounding the Berlin Wall. Therefore, professors should rethink how they design the courses. They should make a shift from purely lecture-based learning towards technologybased learning where students collaborate and come up with creative and novel solutions in a team setting.

\section{CHART RADAR AND ECDIS}

A segment of the analyzed COIL project was the DUT students' attempt to explain to their colleagues at the UoC the basic features of the modern chart radar and ECDIS. Accordingly, the following sub-sections give an overview of these two advanced navigation systems.

Radar is the most important electronic aid for observation and evaluations of the risk of collision. (Satellite-) Automatic Identification System (S-)(AIS) and ECDIS must not be used as aids alone, without comparison with the radar display. Most modern radars can show chart data together with ordinary radar data. This is the so-called chart radar. Chart data on chart radar must be updated in the same way as on ECDIS. Also, it is possible to show radar data on ECDIS. This is called radar overlay, while radar video is transferred directly from the video output port on the electronic chart. Radar is the safest aid to coastal navigation. It is almost unthinkable to leave port without having the radar in order. Seafarers should be capable to interpret the radar display in a fast and safe way. They have to be critical about having too much information on the radar screen since the important small echoes can be easily overlooked. All the synthetic data at frequent intervals are to be removed in order to keep a good radar observation.

The primary function of radar, including chart radar, is collision avoidance. On the other hand, ECDIS' primary function is avoiding grounding. ECDIS is a system that displays hydrographic information, which are combined with information provided by electronic position-fixing systems like Global Positioning System (GPS), radar, Automatic Radar Plotting Aid (ARPA), etc., to assist in the safe navigation of a vessel. It consists of Electronic Navigation Chart (ENC) as a data file, and Electronic Chart Display Equipment (ECDE) hardware (Norris, 2013). In addition, ECDIS is a system which can also store and use information from the list of lights, sailing directions, tide tables, etc., together with the chart. An Electronic Chart System (ECS) is a generic term for equipment which displays electronic charts, but which does not satisfy all Safety of Life at Sea (SOLAS) Convention requirements (SeaGull AS, 2001). ECDIS has made a revolutionary change in the traditional way of navigation during the last decade of the 1990s, and there is a tendency for its full implementation at the global level. The main related problems are numerous non-SOLAS ships (Bauk et al., 2017). Both, (chart) radar and ECDIS should be a perfect tandem for supporting seafarers' decision-making in avoiding collision and grounding respectively.

\section{USABILITY HEURISTICS AND USER INTERFACE DESIGN}

As part of the considered COIL projects, the students at the UoC had the task to explain to the DUT students what is meant by user interface design as part of the analyzed project and how these principles can be applied in the case of integrated navigation systems.

In order to develop applications that are effective, efficient, and easy to use, the developers need to put the user at the center of the development process (Fajardo et al., 2017). Placing users or human elements into the forefront in maritime is a part of IMO resolution $\mathrm{A} .850(20)(\mathrm{IMO}, 2003)$.

The ten heuristics for user interface design by Jakob Nielsen are the most common principles for designing an effective, interactive and attractive software or hardware interface according to the human factors of users (here seafarers). These heuristics are: visibility of the system status, match between the system and the real world, user control and freedom, consistency and standards, error prevention, recognition rather than recall, flexibility and efficiency of use, aesthetic and minimalist design, helping users recognize, diagnose, and recover from errors, and help and documentation (Nielsen, 2014). The related heuristics can be used to evaluate any user interface in order to detect problems and suggest improvements. The heuristics are applied here to assess users' centeredness in the realm of modern chart radar and ECDIS.

\section{APPLIED METHODOLOGY}

The project was carried out during eight weeks in MarchMay, 2019. In the project, twenty-two students from DUT and thirteen students from UoC were involved. Their ages were about twenty and their nationalities were mostly Zulu and Xhosa at DUT, and Mexican at UoC. They were guided by two lecturers: one at DUT, and the other at UoC. Since the South African students were in the field of navigational information systems and the Mexican students were in the domain of software testing methods, they had different tasks. Namely, the South African students' task was to explain to the Mexican students the basis of chart radar and ECDIS, while the Mexican students' task was to apply the ten heuristic principles to assessing user centeredness and friendliness across the mentioned advanced navigational devices' interfaces.

The students were divided into twelve groups, and the teachers provided the lists with the students' names, e-mails, and cell-phone numbers of some students for both the parties 


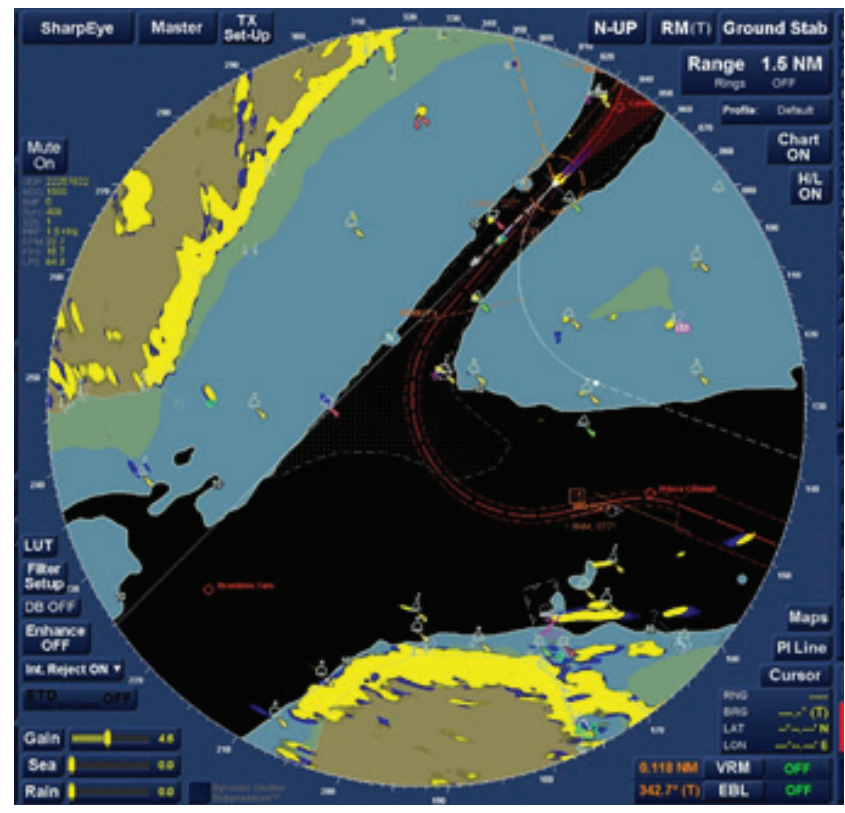

Figure 1.

Chart radar - Kelvin Hughes (Source: Kjerstad (2016), p. 2-77).

involved. Also, the teachers proposed the group leaders at both sides for an initial one-to-one chat. During the ice-breaking Skype session the lecturers explained to the students the project tasks, their roles, and the project goal. They also introduced the students with radar and ECDIS main purposes, functions and key design features on one side, and on the other, with the main principles of ten-heuristics-approach in software design and its testing. When it comes to contemporary navigational devices, different groups of students dealt with different types of navigational equipment interfaces depending on their producers: Kelvin Hughes, (Telchart) Furuno, Selesmar, Simrad, JRC, Maris, Navico, Transas (Wärtsilä), etc.

Afterwards, the students started to chat via mail, WhatsApp, and Skype. In a later phase, they started to work on joint PowerPoint presentations in Google Docs. It was an interesting, dynamic, and edifying process of creating a joint learning environment in the Cloud. The South African students were focused on the main components and purposes of the navigational devices, while the Mexican students were focused on evaluating the interface design according to the heuristics and giving suggestions for improvement. The results of the students' joint work were twelve PowerPoint presentations, whose contents are summarized in the following section. It is important to note that the lecturers from both sides were continuously giving support and directions to the students from both sides. In that regard, in addition to the face-to-face classes at DUT and UoC, several group Skype meetings were organized and realized during the project. It is important to mention that the lecturers and students had to deal with the eight-hour-lag between South Africa and Mexico.
Therefore, group Skype meetings were organized in the morning at UoC, i.e. in the evening at DUT. The lecturers used to arrange their own preparatory Skype meetings in their free time and/or during the weekends.

\section{EVALUATING THE TEN HEURISTICS ON CHART RADAR AND ECDIS}

As stated in the previous section, the heuristic evaluation of some contemporary navigational aids' interface design was performed by the UoC Mexican students, once their DUT South African counterparts had explained them the purposes, basic functions and features of radar in general, with a particular emphasize on chart radar and also on ECDIS. The students were divided in groups and made twelve PowerPoint presentations in Google Docs, out of which their lecturers, i.e., the authors of this paper, have chosen the best insights and summarized them in Tables 1 and 2 on the basis of the analyzed navigational devices' interfaces given in Figures 1 and 2 .

The students from UoC were working, among others, on the Kelvin Hughes chart radar display since their DUT counterparts had explained to them the main purpose, different presentations, i.e. the screen orientation, functions available on the display, and communication/connection between ECDIS and radar. The DUT students had also explained in detail the meaning of the symbols on the chart display. The UoC students' observations regarding the advantages and disadvantages of this type of display from the aspect of its design are summarized in Table 1. 
Table 1.

Chart radar \& ten heuristic principles (Source: Own).

\section{Advantages}

\section{User control and freedom.}

User has freedom to choose different controls. For instance:

TX (transmitting) or RX (receiving); Set-Up: N-UP (north-up),

H-UP (head-up) or C-UP (course-up) display mode; RM (relative motion) or TM (true motion) presentation; Ground Stab (ground stabilization) or Sea Stab (sea stabilization), etc. However, the information related to connected sensors can only be monitored on chart radar display.

If the user has made an error in the radar configuration, s/he can return to the default configuration of each component of the radar and its display.

\section{Match between the system and the real world.}

There is the absolute match in the true motion and relative motion due to the own ship, which is always in the centre of the display in the relative motion. However, the seafarer familiarization with the relevant moving and fixed objects' presentation is of high importance.

Additionally, the chart radar deploys graphical symbols that the seafarer can recognize as boats, buoys, lights, light-houses, pillars, separation schemes, etc. The nuance of sea color can help the navigator to quickly get an overview of the sea depth (e.g., dark blue: shallow water; light blue: deep sea).

\section{Visibility of the system status.}

The system status is always visible, e.g. Variable Range Marker (VRM) and Electronic Bearing Line (EBL) values are given in [m] and $\left[^{\circ}\right]$ respectively; information on chosen maximal Range is visible, as well as information on Gain, Clutter, etc.

However, the screen sometimes indicates a lot of information simultaneously, which can confuse the user. Therefore, the users should know on which information to focus in certain occasions.

Help users to recognize, diagnose and recover from errors The only way in which chart radar can help users in this regard is via audio and/or visual alarms. However, the users, i.e. seafarers, have to be well trained and skillful to diagnose and recover from the error(s).

\section{Recognition rather than recall.}

The user can easily distinguish between the moving and fixed objects.

\section{Disadvantages}

\section{Aesthetic and minimal design.}

Due to the complexity of chart radar it is very difficult to provide a minimalist design. The information is unevenly distributed all over the screen so that there is a minimal order and no clear point of reference. In presenting additional data on the chart radar, the operator must evaluate the need for this critically. Important chart radar data can easily drown in unnecessary text and/or graphical symbols.

\section{Flexibility and efficiency to use.}

The efficient use of chart radar depends mostly on the seafarer's skills. In other words, the seafarers have to adapt to the radar. There is no way round. If one is a beginner in the world of chart radars, s/he will probably have problems to understand the information provided.

\section{Consistency and standards.}

Currently, there is a lack of uniformity and consistency with respect to what information is considered critical and how it should be displayed. For instance, the color of radar objects' presentation on the chart varies with the producer. The names of functions and features including their arrangements may vary as well.

New, harmonized performance standards are to be widely adopted and provide intelligent integration of all relevant information from different sensors.

\section{Error prevention.}

There is no error prevention for the user. The user can not do much when it comes to system error. (S)He must closely observe and follow all the available information from different navigation aids as well as react timely and properly in order to avoid an accident. Chart radar is a monitoring and controlling, but not a handling device. In fact, it is not a maneuvering system, but it can make maneuvering easier.

\section{Help and documentation.}

Chart radar does not give the user any help on the screen if s/he has a problem while doing a certain activity. If something wrong has happened, the chart radar should give the user a good feedback on the error to assist him/her in resolving the problem. There is always an extensive manual onboard with some help instructions, but they are not always user-friendly or practical. 
Another set of observations have been made through the analysis of the students' presentations of Telchart Furuno ECDIS. The South African students explained to their COIL partners from Mexico the main components of the display, including the purpose of tab controls, falling windows, and their key functions (Figure 2).
Upon the explanations given by the South African students, the Mexican students evaluated the design using the ten heuristic principles in order to examine the display from the aspect of its user-centeredness, i.e. easiness of use and, consequently, the reliability of the system. The Mexican students' analyzed and summarized observations are presented in Table 2.

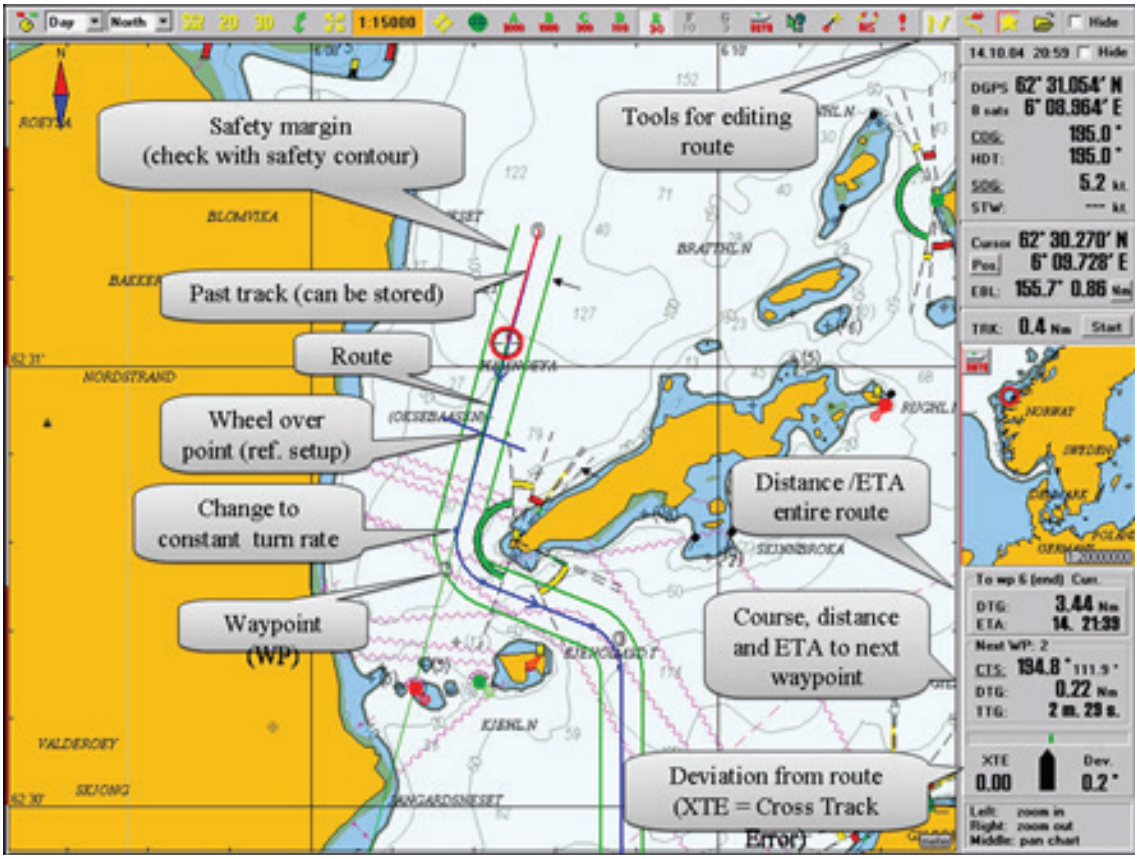

Figure 2.

ECDIS - Telchart Furuno (Source: Kjerstad (2016), p. 2-192).

Table 2

ECDIS \& ten heuristic principles [Source: Own]

\section{Advantages}

\section{User control and freedom.}

User has freedom to choose different control buttons (upper menu, Figure 4) and different falling control panel windows on the left side of the screen. It has to be pointed out that the arrangement of the controls may vary with the producer. Also, the user can divide the chart area into two parts either horizontally or vertically, getting thus a simultaneous insight both into the closest surroundings of the ship and the wider area.

If the user has made an error in adjusting the ECDIS presentation settings, s/he can return to the default presentation mode (standard one) and get quickly an overview of the actual traffic situation.

\section{Disadvantages}

Aesthetic and minimal design.

Due to the complexity of ECDIS, it is very difficult to provide a minimalist design. There are a lot of control buttons, control panel windows, and sometimes at the bottom of the screen there are tabs of functional panels for route planning, monitoring, navigational and safety alarms, etc. In presenting additional data on ECDIS, the operator must critically evaluate the actual need. Important ECDIS data can easily drown in unnecessary text and/or graphical symbols. 


\section{Match between the system and the real world.}

There is absolute match with the position of own ship and surrounding objects in real time. This is not the case with paper charts. However, sometimes a mismatching of radar overlay and chart image might happen, and an experienced user should know how to interpret the distortion.

Additionally, the ECDIS deploys graphical symbols that seafarer can recognize as boats, buoys, lights, lighthouses, separation schemes, etc. The nuance of the sea color can help the navigator to quickly get an overview of sea depth (e.g. dark blue: shallow water; light blue: deep sea).

\section{Visibility of the system status.}

The system status is visible, e.g. ship's longitude and latitude, course and speed over ground, heading, speed through the water, estimated time of arrival, cross track error, etc. Detailed information on each way-point is also available including alteration of course at each waypoint, and alike. Information on wind, current and tide can also be visible. The screen might contain radar and AIS overlay, including all other relevant information for safe and efficient navigation.

\section{Help users to recognize, diagnose and recover from errors} The only way in which ECDIS can help its users in this regard is via audio and/or visual alarms. However, the users, i.e. seafarers, have to be well trained and skillful to diagnose and recover from the error(s).

\section{Recognition rather than recall.}

The user can easily distinguish between moving and fixed objects.

\section{Flexibility and efficiency to use.}

The efficient use of ECDIS mostly depends on the seafarers' skills. In other words, seafarers have to adapt to the ECDIS. There is no way round. If one is a beginner in the world of ECDIS, s/ he will probably have problems to understand the majority of information provided.

\section{Consistency and standards.}

Currently, there is a lack of uniformity and consistency with respect to what information is considered critical and how it should be displayed on ECDIS. For instance, the appearance of the chart display and control panels and/or buttons varies with the producer. The names of functions and features may vary as well.

New, harmonized performance standards are to be widely adopted. They provide intelligent integration of all the relevant information from different sensors.

\section{Error prevention.}

There is no error prevention for the user. The user cannot do much when it comes to a system error. The user must sharply observe and follow all available information from different navigation aids to react timely and properly in order to avoid an accident. ECDIS is a monitoring and controlling, but not a ship-handling system although it makes ship handling or maneuvering much easier.

\section{Help and documentation.}

The ECDIS does not give the user any help on the screen if s/he has a problem while doing a certain activity. If something goes wrong, ECDIS should give the user a good feedback of error at the display to assist him/her to resolve the problem. However, there are always the manuals on board with plenty of instructions, but sometimes they are not practical or user friendly.
The following section highlights the observed advantages and disadvantages of the analyzed interfaces of particular types of modern navigational aids, i.e. chart radar and ECDIS, with the intention to give the equipment designers at least an idea about the users', i.e. seafarers' real needs and preferences/expectations when it comes to the display design and available key controls and options.

\subsection{Discussion of the Observations}

By analyzing the identified advantages and disadvantages of the chart radar and ECDIS displays, due to the ten heuristic principles it can be observed that both analyzed navigational aids satisfy or not the same principles. Namely, in both cases the following have been identified as advantages: presence of user control and freedom; match between the system and the real world; visibility of the system status; ability to recognize, diagnose, and recover from error; feature of recognition rather than recall. On the other hand, the following have been identified as disadvantages: lack of aesthetic and minimal design; flexibility and efficiency of use; consistency and standards; error prevention possibilities; including help and documentation on the screen, which are missing.

Since we would like the designers to concentrate more on the drawbacks of the contemporary navigational devices in the future, we have highlighted some of the key problems noticed that should be overcome. When it comes to both chart radar and ECDIS, the following deserve a further, more rigorous investigation that should lead to systemic improvements in the future: 
- (a) Important chart radar and ECDIS data can easily drown in unnecessary text and/or graphical symbols;

- (b) Differences in presentations at different types of chart radar and ECDIS are considerable, depending on their producers. This should be facilitated or the navigators will need specialized training for a particular type of equipment;

(c) Furthermore, the color of radar (chart) objects and presentations on the chart (radar) vary with the producer. For example, the color used to represent water is not always blue across different radars. Sometimes it is black. The same is with the names of functions and features, including their arrangements on the screen. This should also be harmonized in the future to make the seafarer's job easier and safer;

(d) There is no error prevention for the user. User cannot actually do anything with the system errors. Sometimes it is difficult to identify them. Therefore, more safety checks and options, including the appropriate alarms, should be conceived of and fitted;

(e) Neither chart radar nor ECDIS give the user any help on the screen if the seafarer has a problem while doing a certain activity. So, this should also be improved in the future.

Besides these specific recommendations for the designers, in order to improve seafarers' satisfaction and confidence while using these devices, some additional, more general conclusions regarding both collaborative international online learning and navigational equipment interface design are given in the last section.

\section{CONCLUSION}

This article describes the possibility of matching two disciplines, i.e. navigational information systems (DUT, South Africa) and software testing methods (UoC, Mexico), thanks to the attempts of the involved students and lecturers to attract the attention of the architects of modern navigational aids regarding the need to make these devices more user friendly, more reliable and, consequently, safer. The designers of today's navigational systems should not compete among themselves in terms of which system will be more complex and shiny, but which one would be more user friendly, effective, efficient, and safe.

The chart radar and ECDIS systems must be easy to operate and safe to use. The human machine interface and user friendliness of the earlier systems of this kind is not what the mariners want and are likely to accept today. The technology for improvement is here, but the manufacturers have to invest more effort and money to make the contemporary navigation aids more user-friendly and improve its operational safety. Navigators hope and believe that future chart radars and ECDIS systems will be more user-friendly than they are today. The manufacturers should offer additional options if they are actually useful and can be fitted without confusing the operator. The key to designing interfaces that are effective and easy to use is to focus on the users' context, needs, and motivations.

In addition, the article can be also used as an experimental proof of the below listed usual COIL projects' benefits and impediments. Namely, the common benefits of the COIL projects are: students and staff development, interdisciplinary international and professional collaboration, conversations that lead to other forms of collaboration and engagement, etc. On the other hand, general shortages of the COIL projects are: different time zones, languages, institutional cultures and expectations, academic semester time schedules and requirements, course contents, assessment of learning, quality assurance systems, technological issues, lack of technical and administrative support, and alike. However, both advantages and disadvantages should be taken into consideration and used for paving the way for a further development and achievements within the global electronic classrooms of the future.

\section{ACKNOWLEDGEMENT}

We would like to thank the students at the DUT (South Africa) and the UoC (Mexico) for their efforts to accomplish the tasks within the COIL project, and also for their diligence, although they may not agree with all of the interpretations and conclusions of this paper.

\section{REFERENCES}

Anderson, P. et al., 2010. Peer Reviewing Across the Atlantic: Patterns and Trends in L1 and L2 Comments Made in an Asynchronous Online Collaborative Learning Exchange Between Technical Communication Students in Sweden and in the United States. Journal of Business and Technical Communication, 24(3), pp.296-322. Available at:

http://dx.doi.org/10.1177/1050651910363270.

Bauk, S., 2019. Collaborative Online International Learning Benefits Vis-A-Vis Concerns: An Empirical Study. Montenegrin Journal of Economics, 14 (2), pp. 207216

Bauk, S. \& Radlinger, R., 2013. Concerning Web-based e-learning at a Maritime Higher Education Institution: Case Study. Transactions on Maritime Science, 2(2), pp.115-122. Available at: http://dx.doi.org/10.7225/toms.v02.n02.004.

Bauk, S., Kapidani, N., Schmeink, A., (2017). On Intelligent Use of ICT in Some Maritime Business Organizations. Montenegrin Journal of Economics, 13(2), pp. 163-173.

Brendel, W. \& Cornett-Murtada, V., 2018. Professors Practicing Mindfulness: An Action Research Study on Transformed Teaching, Research, and Service. Journal of Transformative Education, 17(1), pp.4-23. Available at: http://dx.doi.org/10.1177/1541344618762535.

Brooks, C. F., 2011. Cross-cultural classroom conversation online: Preparing students for global citizenry. Proceedings of the Society for Information Technology and Teacher Education (SITE) International Conference, pp. 174-179. 
Brooks, C. F., 2012. Considering curriculum and pedagogy: Preparing students for living in an eSociety through cross-cultural conversation in a global classroom. Proceedings of Global Learn, pp. 126-129.

Brooks, C.F. \& Pitts, M.J., 2015. Communication and identity management in a globally-connected classroom: An online international and intercultural learning experience. Journal of International and Intercultural Communication, 9(1), pp.5268. Available at:

http://dx.doi.org/10.1080/17513057.2016.1120849.

EfficientSea, 2.0 Getting Connected, EU H2020 Project No. 636329, 2018. D1.8 Final Usability Report. Available at: https://efficiensea2.org/wp-content/ uploads/2018/04/Deeliverable-1.8-Part-1-final-usability-report-and-fiveappendices-part-I.pdf.

Fajardo-Flores, S., Gaytán-Lugo, L.S. \& Naftali, M., 2017. Usability techniques. Proceedings of the 8th Latin American Conference on Human-Computer Interaction - CLIHC '17. Available at:

http://dx.doi.org/10.1145/3151470.3156651.

IMO, 2003. Role of Human Element, Human ElementVision and Strategy. Submitted by US, Available at: https://www.transportstyrelsen.se/contentassets/87acf213bfef 4ecab11950fcb725e787/77-17-3.pdf.

Hamilton, E.R., Rosenberg, J.M. \& Akcaoglu, M., 2016. The Substitution Augmentation Modification Redefinition (SAMR) Model: a Critical Review and Suggestions for its Use. TechTrends, 60(5), pp.433-441. Available at:

http://dx.doi.org/10.1007/s11528-016-0091-y.

Jahnke, I., 2020. Technology in higher education: learning with it instead of from it Mizzou News, University of Missouri. Available at: https://news.missouri.edu/2020/ technology-in-higher-education-learning-with-it-instead-of-from-it/.
Kjerstad, N., 2016. Electronic and Acoustic Navigation Systems for Maritime Studies, 1st Ed., NTNU - Norwegian University of Science and Technology, Alesund, Norway.

Koehler, M.J. et al., 2013. The Technological Pedagogical Content Knowledge Framework. Handbook of Research on Educational Communications and Technology, pp.101-111. Available at:

http://dx.doi.org/10.1007/978-1-4614-3185-5_9.

Nielesen, J., 1994. 10 Usability Heuristics for User Interface Design. World Leaders in Research-Based User Experience. Available at: https://www.nngroup.com/articles/ ten-usability-heuristics/.

Norris, A., 2013. Integrated Bridge Systems Vol. 2: ECDIS and Positioning, The Nautical Institute, London, UK.

O'Connor, S. et al., 2018. Social media in nursing and midwifery education: A mixed study systematic review. Journal of Advanced Nursing, 74(10), pp.2273-2289. Available at:

http://dx.doi.org/10.1111/jan.13799.

Pillay, S., Samuels, L., 2016. Collaborative Online International Learning (COIL) at Durban University of Technology. The 5th Learning, Teaching and Assessment Symposium, Durban University of Technology, Durban.

Puentedura, R., 2006. Transformation, technology, and education (Blog post). Available at: http://hippasus.com/resources/tte/.

Seagull, AS, CBT ECDIS, Seagull, AS with Made with Macromedia, C-MAP Norway, and Kongsberg Norcontrol, Version A1 (audio \& video instruction material). Horten, Norway, 2001. 